\title{
On the \\ Capillary Surface in a Wide Circular Tube \\ E. Miersemann
}

Dedicated to the memory of Paul Günther

\begin{abstract}
An explicit asymptotic formula for the wide circular tube is proved. The leading term defines the capillary surface over the half plane with the given boundary contact angle. An explicit estimate of the remainder uniform with respect to the boundary contact angle and the radius of the tube is given. This uniform behaviour is caused by the strong non-linearity of the problem and has no counterpart for linear problems. The proof of the main result is based on a maximum principle and on a mapping which brings the right parameter of development onto the right place of the equation.
\end{abstract}

Keywords: Capillarity, circular tubes, asymptotic expansions

AMS subject classification: Primary 76 B 45, secondary 41 A 60, 35 J 70

\section{Introduction and main results}

The equilibrium free surface $S: u=u\left(x_{1}, x_{2}\right)$ of a liquid inside a circular tube of radius $R \in(0, \infty)$ satisfies (see Finn [3: Chapter 1]) the nonlinear elliptic equation

$$
\operatorname{div} T u=\kappa u+\lambda
$$

in the disk $B_{R}=\left\{x=\left(x_{1}, x_{2}\right) \in \mathbb{R}^{2}: x_{1}^{2}+x_{2}^{2}<R_{1}^{2}\right\}$ and the boundary condition

$$
\nu \cdot T u=\cos \gamma \quad \text { on } \partial B_{R}
$$

where

$$
T \dot{u}=\frac{\nabla u}{\sqrt{1+|\nabla u|^{2}}},
$$

and $\kappa=\frac{\rho g}{\sigma}$ (with $\rho$ the density change across free surface, $g$ the gravitational acceleration and $\sigma$ the surface tension) is the (positive) capillary constant. It is assumed that the gravity field is non-zero and directed downward. Further, $\gamma \in[0, \pi]$ denotes the constant contact angle between the capillary surface and the cylinder. The vector $\nu$ is the exterior unit normal on $\partial B_{R}$ in the $\left(x_{1}, x_{2}\right)$-plane and $\lambda$ denotes a Lagrange parameter.

E. Miersemann: Universität Leipzig, Mathematisches Institut, Augustusplatz 10/11, D - 04109 Leipzig

ISSN 0232-2064 / \$2.50 C Heldermann Verlag Berlin 
When the lower end of the tube is immersed in an infinite reservoir, then $\lambda=0$, and if the tube is closed at the lower end by the base domain $B_{R}$, then the Lagrange parameter is defined by

$$
\lambda=\frac{1}{\left|B_{R}\right|}\left(\left|\partial B_{R}\right| \cos \gamma-\kappa V\right),
$$

where $V$ is the given volume of the fluid (see Finn [3: Chapter 1]) provided there are no dry spots on the bottom.

The transformation

$$
u=v-\frac{\lambda}{\kappa}
$$

converts the above equation and boundary condition into the problem

$$
\begin{array}{ll}
\operatorname{div} T v=\kappa v & \text { in } \quad B_{R} \\
\nu \cdot T v=\cos \gamma & \text { on } \quad \partial B_{R} .
\end{array}
$$

We can assume that

$$
0 \leq \gamma \leq \frac{\pi}{2}
$$

holds. The case $\gamma \geq \frac{\pi}{2}$ reduces to that one under the transformation $v \mapsto-v$.

No explicit solution of problem (1.1), (1.2) is known except $u \equiv 0$ in the case $\gamma=\frac{\pi}{2}$. The existence of a radially symmetric solution $w \in C^{\infty}$ in the open unit disk $B_{1}$ was shown by Johnson and Perko [6]. The comparison principle of Concus and Finn [2] implies that this symmetric solution is the only one. Existence of a solution, not only in the case of the capillary tube, follows from a more general argument (see Finn [3: Chapter 7]).

Since $v$ is radially symmetric, the problem (1.1), (1.2) reduces to that to find a function $w=w(r), r=\sqrt{x_{1}^{2}+x_{2}^{2}}$, such that

$$
\left(\frac{r w^{\prime}}{\sqrt{1+w^{\prime 2}}}\right)^{\prime}=\kappa r w \quad \text { for } 0<r<R
$$

and

$$
\lim _{r \rightarrow R} \frac{w^{\prime}}{\sqrt{1+w^{\prime 2}}}=\cos \gamma \quad \text { and } \quad w^{\prime}(0)=0 .
$$

In 1806 Laplace [8] obtained a formal approximation for the height rise of the capillary surface. For example, he calculated an expression for the height $u_{0}$ at the center of a (narrow) circular tube of radius $R$, namely

$$
u_{0} \approx \frac{2 \cos \gamma}{\kappa R}-\left(\frac{1}{\cos \gamma}-\frac{2}{3} \frac{1-\sin ^{3} \gamma}{\cos ^{3} \gamma}\right) R
$$

The first proof of asymptotic correctness of this formula as $R \rightarrow 0$ was given by Siegel [14]. Then, Finn [4] and Siegel [15] obtained explicit bounds that hold throughout the trajectory, and are not merely asymptotic. The existence of the complete asymptotic 
expansion of the solution to problem (1.1), (1.2) or, equivalently, to problem (1.3), (1.4) as $\sqrt{\kappa} R \rightarrow 0$ was proved by Miersemann [9]. This expansion is uniform with respect to the boundary contact angle despite the fact that $|\nabla w|$ becomes unbounded if $\gamma \rightarrow 0$ or $\gamma \rightarrow \pi$. The reason for this behaviour is the strong non-linearity of the problem. Such expansions exist also for capillary tubes with more general cross sections (see Miersemann [10]), in particular for annular domains.

The above asymptotic formula makes no sense for large radius $R$. On the other hand, by matching asymptotic expansions on adjoining annular subdomains one obtains a comparison surface for the problem over the entire domain also for large $R$. This consideration leads to a new numerical method for solving the capillary tube problem. Moreover, an explicit a priori error estimate uniform with respect to the boundary contact angle can be shown (see Miersemann [11]). This uniform behaviour is caused by the strong non-linearity of the problem and has no counterpart for linear problems. This method applied to a disk and an adjoining annular domain leads to a boundarylayer approximation of the solution. We will not discuss this method in this note.

A formal asymptotic solution of problem (1.3), (1.4) for large $\sqrt{\kappa} R$ was calculated by Concus [1] by using a boundary-layer technique which goes back to Laplace [8]. The idea is to assume that there is a central core region covering most of the base domain in which $w$ is small, and a boundary layer region near the wall in which $w^{\prime}$ increases rapidly to its given boundary value. Matching the core and the boundary-layer solutions in the transition circle determines the thickness of the boundary layer. This method was used by Perko [12] to prove that a certain boundary-layer approximation is assymptotically correct. More precisely, it is shown that for each given boundary contact angle $\gamma$ away from the critical angle $\gamma=0$ the relative error in the ordinate and slope of this boundarylayer approximation is uniform of order $\frac{1}{R} \ln \frac{1}{R}$ as $R \rightarrow \infty$ for $0<r<R$. A formal second order boundary-layer approximation was calculated by Rayleigh [13].

It was shown by Siegel [14] that near the boundary the solution approaches the one-dimensional solution defined by formula (1.8) below. We will prove that there is an asymptotic expansion in powers of $\frac{1}{\sqrt{\kappa} R}$ as $\sqrt{\kappa} R \rightarrow \infty$. This result is of interest near the boundary. Away from the boundary there is a much faster exponental decay (see Siegel [14]).

Let $w(r ; R, \gamma, \kappa)$ be the solution of the boundary value problem (1.3), (1.4) and $v(s ; \gamma, \kappa)$ the solution of the following boundary value problem (1.6), (1.7), which defines the capillary surface over a half plane with the same boundary contact angle. We will prove, in particular, that

$$
|w(r ; R, \gamma, \kappa)-v(R-r ; \gamma, \kappa)| \leq 2.1\left|\frac{\pi}{2}-\gamma\right| \frac{1}{\kappa R}
$$

uniformly in $r \in[0, R]$ and $\gamma \in[0, \pi]$, provided that $\sqrt{\kappa} R \geq 6.4$. This uniformity in $\gamma$ is caused by the strong non-linearity of the problem. Thus, one can use this formula for measurements, for example of the unknown boundary contact angle.

The function $v(s) \equiv v(s ; \gamma, \kappa)$ is defined by the solution of the boundary value problem (1.1), (1.2), where $B_{R}$ has to be replaced by the half plane $\Omega \doteq\left\{\left(x_{1}, x_{2}\right) \in\right.$ 
$\left.\mathbb{R}^{2}: x_{1}>0\right\}$. The assumption that the solution of this problem depends on $x_{1}$ only leads to the boundary value problem

$$
\left(\frac{v^{\prime}(s)}{\sqrt{1+v^{\prime}(s)^{2}}}\right)^{\prime}=\kappa v(s) \quad \text { for } 0<s<\infty
$$

and

$$
\lim _{s \rightarrow 0} \frac{v^{\prime}(s)}{\sqrt{1+v^{\prime}(s)^{2}}}=\cos \gamma .
$$

Under the growth assumptions $v \rightarrow 0$ and $v^{\prime} \rightarrow 0$ as 's $\rightarrow \infty$ one obtains the well-known solution $v \equiv v(s)$ defined by

$$
s=\frac{1}{\sqrt{\kappa}} \ln \left(\frac{\left(\frac{2}{\sqrt{\kappa}}-\sqrt{\frac{4}{\kappa}-v^{2}}\right) h}{\left(\frac{2}{\sqrt{\kappa}}-\sqrt{\frac{4}{\kappa}-h^{2}}\right) v}\right)+\sqrt{\frac{4}{\kappa}-v^{2}}-\sqrt{\frac{4}{\kappa}-h^{2}}
$$

where

$$
h=v(0)=\sqrt{\frac{2}{\kappa}} \sqrt{1-\sin \gamma}
$$

defines the rise of the fluid at the vertical wall. From the comparison principle of Concus and Finn in the case of unbounded domains (see Finn and Hwang [5]) it follows that this solution is the unique solution of the original problem (1.2) over the half plane $\Omega$. We emphasize that for this result one makes neither the above growth assumptions nor the assumption that the solution is independent of $x_{2}$.

Inequality (1.5) and (1.9) yield an explicit estimate for the rise $w_{1} \equiv w(R ; R, \gamma, \kappa)$ of the capillary surface on the boundary of the tube:

$$
\left|w_{1}-\sqrt{\frac{2}{\kappa}} \sqrt{1-\sin \gamma}\right| \leq 2.1\left|\frac{\pi}{2}-\gamma\right| \frac{1}{\kappa R},
$$

uniformly in $\gamma \in[0, \pi]$, provided that $\sqrt{\kappa} R \geq 6.4$. In the case of water $\left(20^{\circ} \mathrm{C}\right)$ in an acrylic plastic tube with radius $R[\mathrm{~cm}]$ one has approximately $\kappa=134\left[\mathrm{~cm}^{-2}\right]$ and $\gamma=80^{\circ}$. Thus the outer height $w_{1}[\mathrm{~cm}]$ is bounded by $\left|w_{1}-0.015\right| \leq \frac{0.003}{R}$, when $R \geq 0.56[\mathrm{~cm}]$.

The proof of the above asymptotic formula (1.5). is based on the comparison principle of Concus and Finn and on the construction of an approximate solution in the sense of the following section. The method is, in principle, the same one we used in Miersemann [9], where the existence of a complete asymptotic expansion as $\sqrt{\kappa} R \rightarrow 0$ was shown. The additional idea is now to map the boundary $r=R$, where the surface rises much more then in regions far from the boundary, onto the origin. This transformation brings the right parameter of expansion onto the right place of the equation (sce formula (2.4) below). This method leads also to a complete asymptotic expansion (see the related remarks in the next section). For simplicity, we restrict ourselves to the leading term given by $v$ (see formula (1.5)).

Combining a comparison principle of Siegel [14] for domains and the above boundary estimates for the circular tube, one obtains a boundary estimate for general domains (see Siegel [14]): 
Let $\Omega \subset \mathbb{R}^{2}$ and $u$ be a solution of $\operatorname{div} T u=\kappa u$ in $\Omega$ and $\nu \cdot T u=\cos \gamma$ on $\partial \Omega$. By $u^{i}$ we denote solutions of the same boundary value problem over a disk $D_{R} \subset \Omega$. If $\Omega$ satisfies a uniform inner sphere condition with radius $R>0$, then $u^{i}>u$ in $D_{R}$.

From this theorem and the estimate (1.10) there follows immediately the explicit estimate

$$
\max _{\partial \Omega} u \leq \sqrt{\frac{2}{\kappa}} \sqrt{1-\sin \gamma}+2.1\left(\frac{\pi}{2}-\gamma\right) \frac{1}{\kappa R}
$$

where $0 \leq \gamma \leq \frac{\pi}{2}$, provided that $\sqrt{\kappa} R \geq 6.4$. From another comparison principle for domains (sce Finn [3: p. 122]) one obtains the lower bound

$$
\min _{\partial \Omega} u \geq \sqrt{\frac{2}{\kappa}} \sqrt{1-\sin \gamma}
$$

under the assumption that $\Omega$ is convex. This follows by comparison of the solution over $\Omega$ with solutions over half planes which contact $\Omega$ at boundary points.

\section{Proof of the asymptotic formula}

Instead of problem (1.3), (1.4) we consider the normalized problem

$$
\begin{aligned}
& \left(\frac{\rho u^{\prime}(\rho)}{\sqrt{1+u^{\prime}(\rho)^{2}}}\right)^{\prime}=\rho u(\rho) \quad \text { for } 0<\rho<M \\
& \lim _{\rho \rightarrow M} \frac{u^{\prime}(\rho)}{\sqrt{1+u^{\prime}(\rho)^{2}}}=\cos \gamma \text { and } u^{\prime}(0)=0 .
\end{aligned}
$$

These problems are related through the formulas

$$
M=\sqrt{\kappa} R, \quad \rho=\sqrt{\kappa} r, \quad u(\rho)=\sqrt{\kappa} w(r) .
$$

Let $u(\rho ; M, \gamma)$ be the solution to problem $(2.1),(2.2)$. Then $v(s)=u(M-s ; M, \gamma)$ solves the boundary value problem

$$
\begin{aligned}
\frac{1}{M-s}( & \left(\frac{(M-s) v^{\prime}(s)}{\sqrt{1+v^{\prime}(s)^{2}}}\right)^{\prime}=v(s) \quad \text { for } 0<s<M \\
& \lim _{s \rightarrow 0} \frac{v^{\prime}(s)}{\sqrt{1+v^{\prime}(s)^{2}}}=-\cos \gamma \quad \text { and }{ }^{\prime} \quad v^{\prime}(M)=0 .
\end{aligned}
$$

This boundary value problem becomes singular if $s=M$.

Definition. A function $v_{n}(s)=v_{n}(s ; M, \gamma)$ is said to be an approximate solution to problem (2.4), (2.5) of order $n$ if

$$
\text { (i) } \frac{1}{M-s}\left(\frac{(M-s) v_{n}^{\prime}(s)}{\sqrt{1+v_{n}^{\prime}(s)^{2}}}\right)^{\prime}-v_{n}(s)=R_{n+1}
$$


where $\left|R_{n+1}\right| \leq \frac{c_{n+1}}{M^{n+1}}$ with a constant $c_{n+1}$ independent of $M \in\left[M_{0}, \infty\right)$ with $M_{0}>0$ and of $\gamma \in\left[0, \frac{\pi}{2}\right]$ and

(ii) $\lim _{s \rightarrow 0} \frac{v_{n}^{\prime}(s)}{\sqrt{1+v_{n}^{\prime}(s)^{2}}}=-\cos \gamma$ and $v_{n}^{\prime}(M)=0$.

Note. The crucial point here is that the estimate of $R_{n+1}$ is uniform with respect to $\gamma$ and $M$.

The formal Ansatz

$$
v_{n}(s ; M, \gamma)=\sum_{k=0}^{n} \phi_{k}(s ; M, \gamma) \frac{1}{M^{k}}
$$

and the equation

$$
\frac{1}{M-s}\left(\frac{(M-s) v_{n}^{\prime}(s)}{\sqrt{1+v_{n}^{\prime}(s)^{2}}}\right)^{\prime}-v_{n}(s)=O\left(\frac{1}{M^{n+1}}\right)
$$

with the boundary conditions

$$
\lim _{s \rightarrow 0} \frac{v_{n}^{\prime}(s)}{\sqrt{1+v_{n}^{\prime}(s)^{2}}}=-\cos \gamma \quad \text { and } \quad v_{n}^{\prime}(M)=0
$$

lead to a recurrent system of one nonlinear boundary value problem for $\phi_{0}$ and $n$ linear boundary value problems for $\phi_{k}(k=1, \ldots, n)$ :

The leading term $\phi_{0}$ is defined by the boundary valuc problem (2.6) and (2.7) below, $\phi_{1}$ is the solution of

$$
\left(\frac{\phi_{1}^{\prime}}{\left(1+\phi_{0}^{\prime 2}\right)^{\frac{3}{2}}}\right)^{\prime}-\phi_{1}=\frac{1}{1-\frac{s}{M}} \frac{\phi_{0}^{\prime}}{\sqrt{1+\phi_{0}^{\prime 2}}} \quad \text { for } 0<s<M
$$

with the boundary conditions

$$
\lim _{s \rightarrow 0} \frac{\phi_{1}^{\prime}}{\left(1+\phi_{0}^{\prime 2}\right)^{\frac{3}{2}}}=0 \quad \text { and } \quad \phi_{1}^{\prime}(M)=0
$$

and $\phi_{2}$ is defined by the boundary value problem

$$
\left(\frac{\phi_{2}^{\prime}}{\left(1+\phi_{0}^{\prime 2}\right)^{\frac{3}{2}}}\right)^{\prime}-\phi_{2}=\frac{1}{1-\frac{3}{M}} \frac{\phi_{1}^{\prime}}{\left(1+\phi_{0}^{\prime 2}\right)^{\frac{3}{2}}}-\left(f_{2}\left(\phi_{0}^{\prime}, \phi_{1}^{\prime}\right)\right)^{\prime} \quad \text { for } 0<s<M
$$

with the boundary conditions

$$
\lim _{s \rightarrow 0} \frac{\phi_{2}^{\prime}}{\left(1+\phi_{0}^{\prime 2}\right)^{\frac{3}{2}}}=\ddot{0} \quad \text { and } \quad \phi_{2}^{\prime}(M)=0 .
$$


When $k \geq 3$, then $\phi_{k}$ is given by

$$
\begin{aligned}
\left(\frac{\phi_{k}^{\prime}}{\left(1+\phi_{0}^{\prime 2}\right)^{\frac{3}{2}}}\right)^{\prime}-\phi_{k}= & \frac{1}{1-\frac{s}{M}}\left(\frac{\phi_{k-1}^{\prime}}{\left(1+\phi_{0}^{\prime 2}\right)^{\frac{3}{2}}}+f_{k-1}\left(\phi_{0}^{\prime}, \ldots, \phi_{k-2}^{\prime}\right)\right) \\
& -\left(f_{k}\left(\phi_{0}^{\prime}, \ldots, \phi_{k-1}^{\prime}\right)\right)^{\prime} \quad \text { for } 0<s<M
\end{aligned}
$$

with the boundary conditions

$$
\lim _{s \rightarrow 0} \frac{\phi_{k}^{\prime}}{\left(1+\phi_{0}^{\prime 2}\right)^{\frac{3}{2}}}=0 \quad \text { and } \quad \phi_{k}^{\prime}(M)=0
$$

The functions

$$
f_{j}=f_{j}\left(\phi_{0}^{\prime}, \ldots, \phi_{j-1}^{\prime}\right)
$$

are analytic in their arguments and satisfy $f_{j}\left(y_{0}, \ldots, y_{j-1}\right)=0$ if one of the coordinates $y_{l}$ vanishes. These functions $f_{j}(2 \leq j \leq n)$ are defined by the identity

$$
\begin{aligned}
\frac{\sum_{l=0}^{n} y_{l} \mu^{l}}{\sqrt{1+\left(\sum_{l=0}^{n} y_{l} \mu^{l}\right)^{2}}}= & \frac{y_{0}}{\sqrt{1+y_{0}^{2}}}+\frac{y_{1}}{\left(1+y_{0}^{2}\right)^{\frac{3}{2}}} \mu \\
& +\sum_{l=2}^{n}\left(\frac{y_{l}}{\left(1+y_{0}\right)^{\frac{3}{2}}}+f_{l}\left(y_{0}, \ldots, y_{l-1}\right)\right) \mu^{l}+O\left(\mu^{n+1}\right)
\end{aligned}
$$

as $\mu \rightarrow 0$. For example, one has

$$
f_{2}=-\frac{3}{2} \frac{y_{0} y_{1}^{2}}{\left(1+y_{0}^{2}\right)^{\frac{3}{2}}} .
$$

From the non-linearity of the problem one concludes that $v_{n}$ defines an approximate solution in the sense of the above definition and that the functions are analytic on the interval $[0, M)$ and bounded on the closed interval. Then the comparison principle of Concus and Finn [2] implies a complete asymptotic expansion of $v$ in powers of $\frac{1}{M}$ (see Miersemann [9] for this method in the case of a narrow tube). To simplify the presentation, we will restrict ourselves to the case $n=0$.

Theorem 2.1. Let $v_{n}(s ; M, \gamma)$ be an approximate solution in the sense of the above definition and let $u(\rho ; M, \gamma)$ be the solution of the boundary value problem (2.1), (2.2). Then

$$
\left|u(\rho ; M, \gamma)-v_{n}(M-\rho ; M, \gamma)\right| \leq \frac{c_{n+1}}{M^{n+1}}
$$

for $\rho \in[0, M]$, where $c_{n+1}$ is the constant in the above definition.

Proof. Set $U_{n}(x)=v_{n}(M-|x| ; M, \gamma)$. Then

$\left|\operatorname{div} T U_{n}-U_{n}\right| \leq \frac{c_{n+1}}{M^{n+1}}$ in $B_{M}(0) \quad$ and $\quad \lim _{|x| \rightarrow M} \nu \cdot T U_{n}=\cos \gamma$ on $\partial B_{M}(0)$. 
Using the comparison function $U^{+}=U_{n}+\frac{c_{n+1}}{M^{n+1}}$, we obtain

$$
\operatorname{div} T U^{+}-U^{+} \leq 0 \text { in } B_{M}(0) \quad \text { and } \quad \lim _{|x| \rightarrow M} \nu \cdot T U^{+}=\cos \gamma \text { on } \partial B_{M}(0) .
$$

Since the function $U(x)=u(|x| ; M, \gamma)$, where $u$ defines the solution of the boundary value problem (2.1), (2.2), satisfies

$$
\operatorname{div} T U-U=0 \text { in } B_{M}(0) \quad \text { and } \quad \lim _{|x| \rightarrow M} \nu \cdot T U=\cos \gamma \text { on } \partial B_{M}(0)
$$

the comparison principle of Concus and Finn [2] implies that $U(x) \leq U^{+}(x)$ in $B_{M}(0)$. Analogously, the comparison function $U^{-}=U_{n}-\frac{c_{n+1}}{M^{n+1}}$ yields a lower bound of $U(x)$ in $B_{M}(0)$

Let $\phi_{0}(s ; M, \gamma)$ be the solution of the boundary value problem

$$
\left(\frac{u^{\prime}}{\sqrt{1+u^{\prime 2}}}\right)^{\prime}=u \quad \text { for } 0<s<M
$$

and

$$
\lim _{s \rightarrow 0} \frac{u^{\prime}}{\sqrt{1+u^{\prime 2}}}=-\cos \gamma \quad \text { and } \quad u^{\prime}(M)=0 .
$$

This solution $\phi_{0}$ determines the capillary surface between two parallel walls with the distance $M$ and the boundary contact angles $\gamma$ and $\frac{\pi}{2}$ on the walls over the line $x_{1}=0$ and $x_{1}=M$, respectively. Set

$$
N(v)=\frac{1}{M-s}\left(\frac{(M-s) v^{\prime}(s)}{\sqrt{1+v^{\prime}(s)^{2}}}\right)^{\prime} .
$$

Then .

$$
N\left(\phi_{0}\right)-\phi_{0}=-\frac{1}{M} \frac{M}{M-s} \frac{\phi_{0}^{\prime}}{\sqrt{1+\phi_{0}^{\prime 2}}} .
$$

The following two lemmas are shown in the Appendix.

Lemma 2.1. The function $\phi_{0}$ satisfies

$$
\frac{M}{M-s} \frac{\left|\phi_{0}^{\prime}(s)\right|}{\sqrt{1+\phi_{0}^{\prime}(s)^{2}}} \leq 2\left|\frac{\pi}{2}-\gamma\right|
$$

uniformly with respect to $s \in(0, M)$ with $M \in[6.4, \infty)$ and to $\gamma \in[0, \pi]$.

Note. An inspection of the proof of this lemma shows that the constant 2 on the right-hand side of the above estimate can be replaced by $1+\varepsilon$ for each $\varepsilon>0$ when $M(\varepsilon) \leq M<\infty$.

Let $\psi_{0}(s ; \gamma)$ be the solution of the boundary value problem (1.6) with $\kappa=1$ and (1.7). This function defines the capillary surface over a half plane (see Section 1). 
Lemma 2.2. The functions $\phi_{0}$ and $\psi_{0}$ satisfy

$$
\left|\phi_{0}(s ; M, \gamma)-\psi_{0}(s ; \gamma)\right| \leq 7.1\left|\frac{\pi}{2}-\gamma\right| e^{-M}
$$

uniformly with respect to $s \in(0, M)$ with $M \in[6.4, \infty)$ and to $\gamma \in[0, \pi]$.

Combining Theorem 2.1, Lemma 2.1 and Lemma 2.2 we are lead to the following

Theorem 2.2. The difference between the solution $u$ of problem (2.1), (2.2) and of the function $\psi_{0}$ which defines the capillary surface over a half plane with the boundary contact angle $\gamma$ can be estimated by

$$
\left|u(\rho ; M, \gamma)-\psi_{0}(M-\rho ; \gamma)\right| \leq 2.1\left|\frac{\pi}{2}-\gamma\right| \frac{1}{M}
$$

uniformly with respect to $\rho \in(0, M)$ with $M \in[6.3, \infty)$ and to $\gamma \in[0, \pi]$.

From the transformation formulas (2.3) one obtains the estimate (1.5) of Section 1 for the solution $w$ of the original problem.

\section{Appendix}

In this section we prove Lemmas 2.1 and 2.2. The solution of the boundary value problem (2.6), (2.7) can be defined by an elliptic integral (see, for example, Landau and Lifschitz [7: p. 272]). We need some explicit estimates on $\phi_{0}$ for the proof of these lemmas. For the convenience of the reader, we will give these easy calculations in this Appendix.

From the identity

$$
\left(\frac{u^{\prime}}{\sqrt{1+u^{\prime 2}}}\right)^{\prime} \equiv-\frac{1}{u^{\prime}}\left(\frac{1}{\sqrt{1+u^{\prime 2}}}\right)^{\prime}
$$

and equation (2.6) there follows

$$
\frac{1}{\sqrt{1+u^{\prime 2}}}=-\frac{1}{2} u^{2}+C
$$

with a constant $C$. The boundary conditions (2.7) imply

$$
\begin{aligned}
1 & =-\frac{1}{2} u(M)^{2}+C \\
\sin \gamma & =-\frac{1}{2} u(0)^{2}+C
\end{aligned}
$$

Since $u(M) \neq 0$ when $\gamma<\frac{\pi}{2}$, there follows

$$
C=C(M, \gamma)>1
$$


We recall that we assume $0 \leq \gamma<\frac{\pi}{2}$ and that the case $-\frac{\pi}{2}<\gamma \leq 0$ reduces to that one under the mapping $u \mapsto-u$. The substitution

$$
\cos \zeta=C-\frac{1}{2} u^{2} \quad\left(0<\zeta<\frac{\pi}{2}\right)
$$

and the boundary conditions (3.2) and (3.3) lead to the parametric representation (see, for example, Landau and Lifschitz [7: p. 271])

$$
\begin{aligned}
& u(\zeta)=\sqrt{2} \sqrt{C-\cos \zeta} \\
& s(\zeta)=\frac{1}{\sqrt{2}} \int_{\zeta}^{\frac{\pi}{2}-\gamma} \frac{\cos \tau}{\sqrt{C-\cos \tau}} d \tau
\end{aligned}
$$

where $0 \leq \zeta \leq \frac{\pi}{2}-\gamma$ and $C=C(M, \gamma)>1$ is the constant from (3.4). The equation

$$
M=\frac{1}{\sqrt{2}} \int_{0}^{\frac{\pi}{2}-\gamma} \frac{\cos \tau}{\sqrt{C-\cos \tau}} d \tau
$$

implies that for fixed $\gamma \in\left[0, \frac{\pi}{2}\right)$ the function $C(M, \gamma)$ is monotonically decreasing with $M \rightarrow \infty$ and

$$
\lim _{M \rightarrow \infty} C(M, \gamma)=1
$$

Then, from (3.2) we obtain $\lim _{M \rightarrow \infty} u(M)=0$.

Lemma 3.1. Set $\delta=\frac{\pi}{2}-\gamma$. For $\dot{\gamma} \in\left[0, \frac{\pi}{2}\right)$ and $M \in(1.3, \infty)$ there exists $a$ constant $b(M, \delta)$ uniformly bounded by $|b(M, \delta)|<0.8$ such that

$$
C(M, \gamma)=1+\frac{\varepsilon^{2}}{2} \quad \text { where } \quad \varepsilon=2 \delta \frac{e^{\delta b(M, \delta)+M}}{e^{2 M}-e^{2 \delta b(M, \delta)}}
$$
Then

Proof. This lemma follows from the following decomposition. Let $\zeta \in\left(0, \frac{\pi}{2}-\gamma\right]$.

$$
\begin{aligned}
& \int_{0}^{\zeta} \frac{\cos \tau}{\sqrt{C-\cos \tau}} d \tau \\
& \quad=\int_{0}^{\zeta} \frac{d \tau}{\sqrt{C-1+\frac{r^{2}}{2}}}+\int_{0}^{\zeta} \frac{\cos \tau-1}{\sqrt{C-\cos \tau}} d \tau \\
& \quad+\int_{0}^{\zeta} \frac{1}{\sqrt{C-1+\frac{\tau^{2}}{2}}} \cdot\left(\frac{1}{1-\frac{\cos \tau-1+\frac{r^{2}}{2}}{C-1+\frac{\tau^{2}}{2}}}-1\right) d \tau
\end{aligned}
$$

This implies

$$
\int_{0}^{\zeta} \frac{\cos \tau}{\sqrt{C-\cos \tau}} d \tau=\int_{0}^{\zeta} \frac{d \tau}{\sqrt{C-1+\frac{\tau^{2}}{2}}}+\sqrt{2} \zeta b(C, \zeta)
$$


where

$$
|b(M, \zeta)|<0.8
$$

uniformly in $\gamma \in\left[0, \frac{\pi}{2}\right)$ and $M \in\left[M_{0}, \infty\right)$ with $M_{0}>0$. Set $C-1=\frac{\varepsilon^{2}}{2}$. Then

$$
\int_{0}^{\zeta} \frac{d \tau}{\sqrt{C-1+\frac{r^{2}}{2}}}=\sqrt{2} \ln \left(\frac{\zeta}{\varepsilon}+\sqrt{1+\left(\frac{\zeta}{\varepsilon}\right)^{2}}\right) .
$$

Thus, this equation, (3.7) and (3.9) conclude the proof

From (3.6) we see that $s(\zeta)$ is strictly increasing when $\zeta$ decreases. Set $\delta=\frac{\pi}{2}-\gamma$ and $\hat{\delta}=\zeta \frac{M}{2}$.

Lemma 3.2. When $M \geq 5.1$, then there exist constants $b(M, \delta)$ and $b(M, \hat{\delta})$ such that

$$
\zeta\left(\frac{M}{2}\right)=\delta e^{-\frac{M}{2}} \frac{e^{\delta b(M, \delta)}\left(1-e^{2 \dot{\delta} b(M, \hat{\delta})-M}\right)}{e^{\hat{\delta} b(M, \hat{\delta})}\left(1-e^{2 \delta b(M, \delta)-2 M}\right)}
$$

and the constants $b$ satisfy inequality (3.10).

Proof. From (3.5) and (3.7) we obtain

$$
\frac{M}{2}=\frac{1}{\sqrt{2}} \int_{0}^{\dot{\delta}} \frac{\cos \tau}{\sqrt{C-\cos \tau}} d \tau
$$

Analogously to the proof of the above Lemma 3.1, we conclude from (3.11) that

$$
\frac{\hat{\delta}}{\varepsilon}=\frac{1}{2} \frac{e^{M}-e^{2 \dot{\delta} b(M, \tilde{\delta})}}{e^{\dot{\delta} b(M, \tilde{\delta})+(M / 2)}}
$$

where $\varepsilon$ is defined through the formula given in Lemma 3.1

Formula (3.5) implies that $u\left(\frac{M}{2}\right)<\varepsilon+\sqrt{2} \zeta\left(\frac{M}{2}\right)$ and together with Lemma 3.1 and Lemma 3.2 we have

$$
u\left(\frac{M}{2}\right) \leq 7.5\left(\frac{\pi}{2}-\gamma\right) e^{-\frac{M}{2}}
$$

Integration of the differential equation (2.6) from $s \in\left(\frac{M}{2}, M\right)$ to $M$ yields

$$
\frac{u^{\prime}(s)}{\sqrt{1+u^{\prime}(s)^{2}}}=-\int_{s}^{M} u d \tau
$$

Thus

$$
\frac{\left|u^{\prime}(s)\right|}{\sqrt{1+u^{\prime}(s)^{2}}} \leq(M-s) u\left(\frac{M}{2}\right) .
$$


Together with (3.12) there follows

$$
\frac{M}{M-s} \frac{\left|u^{\prime}(s)\right|}{\sqrt{1+u^{\prime}(s)^{2}}} \leq 7.5\left(\frac{\pi}{2}-\gamma\right) M e^{-\frac{M}{2}} .
$$

On the other interval $\left(0, \frac{M}{2}\right)$ one has

$$
\frac{M}{M-s} \frac{\left|u^{\prime}(s)\right|}{\sqrt{1+u^{\prime}(s)^{2}}} \leq 2 \frac{\left|u^{\prime}(s)\right|}{\sqrt{1+u^{\prime}(s)^{2}}} \leq 2 \lim _{s \rightarrow 0} \frac{\left|u^{\prime}(s)\right|}{\sqrt{1+u^{\prime}(s)^{2}}}=2 \cos \gamma
$$

since $\frac{\left|u^{\prime}(s)\right|}{\sqrt{1+u^{\prime}(s)^{2}}}$ is strictly increasing when $s \rightarrow 0$. This monotonicity property follows from (3.13) since $u$ is strictly increasing with $s \rightarrow 0$ (see (3.5) and (3.6)).

Proof of Lemma 2.1. The proof follows from (3.14) and (3.15) when $M \geq 6.4$

Proof of Lemma 2.2. We see from (3.5) and Lemma 3.1 that the solution $u$ of the boundary value problem (2.6), (2.7) satisfies

$$
0 \leq u(M) \leq \varepsilon \leq 7.1\left(\frac{\pi}{2}-\gamma\right) e^{-M}
$$

if $M \geq 6.3$. The unique solution $\psi$ of the boundary value problem (1.6), (1.7) over the unbounded interval is defined by (3.5), (3.6) when $C=1$, that is,

$$
\psi(\zeta)=\sqrt{2} \sqrt{1-\cos \zeta} \quad \text { and } \quad s(\zeta)=\frac{1}{\sqrt{2}} \int_{\zeta}^{\frac{\pi}{2}-\gamma} \frac{\cos \tau}{\sqrt{1-\cos \tau}} d \tau
$$

where $0<\zeta \leq \frac{\pi}{2}-\gamma$. Set $\hat{\zeta}=\zeta(M)$. One obtains analogously to the proof of Lemma 3.1

$$
M=\int_{\hat{\zeta}}^{\frac{\pi}{2}-\gamma} \frac{d \tau}{\tau}+c(M, \gamma)\left(\frac{\pi}{2}-\gamma\right)
$$

where $|c(M, \gamma)|<0.8$ uniformly with respect to $M \geq 6.4$ and to $\gamma \in\left[0, \frac{\pi}{2}\right)$. Consequently, $\zeta(M)=\left(\frac{\pi}{2}-\gamma\right) e^{\left(\frac{\pi}{2}-\gamma\right) c(M, \gamma)-M}$ which implies $\zeta(M) \leq 3.6\left(\frac{\pi}{2}-\gamma\right) e^{-M}$. Hence since $\psi(M) \leq \zeta(M)$, one has

$$
0 \leq \psi(M) \leq 3.6\left(\frac{\pi}{2}-\gamma\right) e^{-M}
$$

Finally, one obtains Lemma 2.2 from the comparison principle and (3.16), (3.17)

Acknowledgement. This paper was supported in part by the Deutsche Forschungsgemeinschaft (DFG). 


\section{References}

[1] Concus, P.: Static menisci in a vertical right circular cylinder. J. Fluid Mech. 34 (1968), $481-485$.

[2] Concus, P. and R. Finn: On capillary free surfaces in a gravitational field. Acta Math. 132 (1974), $207-223$.

[3] Finn, R.: Equilibrium Capillary Surfaces. New York: Springer-Verlag 1986.

[4] Finn, R.: On the Laplace formula and the meniscus height for a capillary surface. Z. Angew. Math. Mech. (ZAMM) 61 (1981), 165 - -173.

[5] Finn, R. and J.-F. Hwang: On the comparison principle for capillary surfaces. J. Fac. Sci. Univ. Tokyo 36 (1989), $131-134$.

[6] Johnson, W. E. and L. M. Perko: Interior and exterior boundary value problems from the theory of the capillary tube. Arch. Rat. Mech. Anal. 29 (1968), $125-143$.

[7] Landau, L. D. and E. M. Lifschitz: Hydrodynamik. Berlin: Akademie-Verlag 1974.

[8] Laplace, P. S.: Traité de mécanique céleste, Tome 10/Supplément. Paris: Courcier 1805. In: Oeuvres Complètes de Laplace, Tome 4. Paris: Gauticrs-Villars 1880.

[9] Miersemann, E.: On the Laplace formula for the capillary tube. Asymptotic Anal. 8 (1994), $393-403$.

[10] Miersemann, E.: On the rise height in a capillary tube of general cross section. Asymptotic Anal. 7 (1993), $301-309$.

[11] Miersemann, E.: An asymptotic method for solving the capillary tube problem. Submitted.

[12] Perko, L. M.: Boundary layer analysis of the wide capillary tube. Arch. Rational. Mech. Anal. 45 (1973), 120 - 133.

[13] Lord Rayleigh: On the theory of the capillary tube. Proc. Roy. Soc. London (Ser. A) 92 (1915), $184-195$.

[14] Siegel, D.: Height estimates for capillary surfaces. Pacific J. Math. 88 (1980), $471-516$.

[15] Siegel, D.: Explicit estimates of a symmetric capillary surface for small Bond number. In: Continuum Mechanics and Its Applications (eds.: G. A. C. Graham and S. K. Malik). New York: Hemisphere Publ. Corp. 1989, pp. 497 - 506.

Received 17.05.1996 